\title{
Quotient metrics with applications in convex geometry
}

\author{
Agnieszka Bogdewicz • Irmina Herburt • \\ Maria Moszyńska
}

Received: 12 October 2011 / Accepted: 2 December 2011 / Published online: 15 December 2011

C The Author(s) 2011. This article is published with open access at Springerlink.com

\begin{abstract}
Burago et al. (Graduate studies in mathematics, AMS, 2001) considered quotient metric spaces consisting of orbits with respect to some isometry groups. We extend their approach over some semigroups of transformations. We are concerned with quotient semi-metrics in spaces of generalized orbits and give conditions sufficient for these semi-metrics to be metrics. We apply our approach to hyperspaces of compact convex subsets of Euclidean $n$-space and to that of convex bodies, endowed, respectively, with the Hausdorff metric and with the symmetric difference metric.
\end{abstract}

Keywords Compact convex sets - Convex bodies · Hausdorff metric . Symmetric difference metric · Quotient semi-metric · Quotient metric · Weakly left (right) divisible semigroup

Mathematics Subject Classification (2000) $\quad$ 20M20 $\cdot 54 \mathrm{E} 35 \cdot 54 \mathrm{E} 40 \cdot 54 \mathrm{E} 25$

\section{Introduction}

Burago et al. (2001) considered quotient metric spaces consisting of orbits with respect to some isometry groups. We extend their approach over generalized orbits with respect to some semigroups of transformations.

\footnotetext{
A. Bogdewicz · I. Herburt

Faculty of Mathematics and Information Science, Warsaw University of Technology, pl. Politechniki 1, 00-661 Warsaw, Poland

e-mail: abogde@mini.pw.edu.pl

I. Herburt

e-mail: herbir@mini.pw.edu.pl

M. Moszyńska (ه)

Institute of Mathematics, University of Warsaw, Banacha 2, 02-097 Warsaw, Poland

e-mail: mariamos@mimuw.edu.pl
} 
Section 2 concerns quotient semi-metrics in a rather general setting.

In Sect. 3 we generalize the classical notion of orbit with respect to a group of transformations. Our generalized orbits are defined as the equivalence classes of a relation generated by some semigroups of transformations.

Section 4 concerns quotient semi-metrics induced by some semi-groups of transformations of a given metric space into itself.

In Sect. 5, we give conditions under which a quotient semi-metric is a metric. In particular, we consider the space $\left(\mathcal{K}^{n}, \rho_{H}\right)$ of nonempty compact convex subsets of $\mathrm{R}^{n}$ with the Hausdorff metric and the space $\left(\mathcal{K}_{0}^{n}, \delta\right)$ of convex bodies (members of $\mathcal{K}^{n}$ with nonempty interior) with the symmetric difference metric (see Schneider 1993).

Section 6 deals with Rådström space and the relationship between the metric generated by norm in this space and the quotient metric induced by the "taxi metric" or some other metrics in $\mathcal{K}^{n} \times \mathcal{K}^{n}$ (where $\mathcal{K}^{n}$ is endowed with $\rho_{H}$ ).

\section{Preliminaries}

Following current terminology, for any nonempty set $M$, we refer to a function $\rho: M \times M \rightarrow \mathrm{R}_{+}$as a semi-metric on $M$ if for every $x, y, z \in M$

$$
\rho(x, x)=0, \quad \rho(x, y)=\rho(y, x) \text { and } \rho(x, z) \leq \rho(x, y)+\rho(y, z) .
$$

Thus, a semi-metric $\rho$ is a metric whenever it is non-degenerate:

$$
\rho(x, y)=0 \Longrightarrow x=y \text {. }
$$

Most of examples considered in Sects. 4 and 5 concern the hyperspace $\mathcal{K}^{n}$ of nonempty, compact, convex subsets of $\mathrm{R}^{n}$, with the Hausdorff metric or the hyperspace $\mathcal{K}_{0}^{n}$ of convex bodies (members of $\mathcal{K}^{n}$ with nonempty interiors) with the symmetric difference metric.

The Hausdorff metric $\rho_{H}$ (generally, for compact subsets of any metric space) is defined by

$$
\rho_{H}(X, Y):=\max \left\{\vec{\rho}_{H}(X, Y), \vec{\rho}_{H}(Y, X)\right\}
$$

where

$$
\vec{\rho}_{H}(X, Y):=\sup _{y \in Y} \inf _{x \in X} \rho(x, y),
$$

or equivalently (for $\mathcal{K}^{n}$ )

$$
\vec{\rho}_{H}(X, Y)=\inf \left\{\epsilon>0 \mid X \subset Y+\epsilon B^{n}\right\},
$$

for $B^{n}$ being the Euclidean unit ball in $\mathrm{R}^{n}$ and + being Minkowski addition. 
It is well known that for any $X, Y \in \mathcal{K}^{n}$

$$
\rho_{H}(X, Y)=\sup _{u \in S^{n-1}}|h(X, u)-h(Y, u)|,
$$

where $S^{n-1}$ is the unit sphere in $\mathrm{R}^{n}$ and $h: \mathcal{K}^{n} \times S^{n-1} \rightarrow \mathrm{R}$ is the support function.

The symmetric difference metric $\delta$ is defined by the formula

$$
\delta(X, Y):=V_{n}((X \cup Y) \backslash(X \cap Y))
$$

for any $X, Y \in \mathcal{K}_{0}^{n}$, where $V_{n}$ is $n$-dimensional Lebesgue measure.

In what follows, for any metric space $(M, \rho)$ a map $f: M \rightarrow M$ that preserves metric is referred to as an isometric embedding. An isometric embedding is called an isometry whenever it is surjective.

A map $f: M \rightarrow M$ is a weak contraction if $\rho(f(x), f(y)) \leq \rho(x, y)$ for every $x, y \in M$; it is a weak expansion (or is weakly expanding) if $\rho(f(x), f(y) \geq \rho(x, y)$ for every $x, y \in M$.

\section{Quotient semi-metrics}

Consider a nonempty set $M$ and a function $\rho: M \times M \rightarrow \mathrm{R}_{+}$. For any equivalence relation $\equiv$ on $M$, let $M / \equiv$ be the quotient set. Then $\rho$ induces the function $\hat{\rho}: M / \equiv \times M / \equiv \rightarrow \mathrm{R}_{+}$defined as follows:

Definition 2.1 For equivalence classes $[x]$, $[y]$ of $x, y \in M$ with respect to $\equiv$,

$$
\begin{aligned}
\hat{\rho}([x],[y]):=\inf \left\{\sum_{i=1}^{m} \rho\left(p_{i}, q_{i}\right) \mid p_{1} \equiv x, q_{m} \equiv y,\right. \\
\\
\left.\qquad q_{i} \equiv p_{i+1} \text { for } i=1, \ldots, m-1, m \in \mathrm{N}\right\} .
\end{aligned}
$$

This function $\hat{\rho}$ is related to the semi-metric that was defined in Burago et al. (2001), but our approach is slightly different.

The following is evident.

Proposition 2.2 If for every $x, y \in M$

$$
\rho(x, x)=0 \text { and } \rho(x, y)=\rho(y, x),
$$

then the function $\hat{\rho}$ is a semi-metric in $M / \equiv$; moreover, the quotient map is a weak contraction, i.e.

$$
\hat{\rho}([x],[y]) \leq \rho(x, y)
$$

for every $x, y \in M$.

Remark 2.3 Let $\rho$ be a metric. Then

(i) if $\hat{\rho}$ is a metric, then the equivalence classes of $\equiv$ are closed in $M$; 
(ii) even if the equivalence classes are compact, $\hat{\rho}$ need not be a metric; for instance, this is the case if

$$
M:=\left\{\left(0, \frac{1}{k}\right) \mid k \in \mathrm{N}\right\} \cup\left\{\left(1, \frac{1}{k}\right) \mid k \in \mathrm{N}\right\} \cup\{(0,0),(1,0)\} \subset \mathrm{R}^{2}
$$

with Euclidean metric and the equivalence relation is defined by

$$
\left(x_{1}, x_{2}\right) \equiv\left(y_{1}, y_{2}\right): \Longleftrightarrow\left(x_{1}=y_{1}, x_{2}=y_{2}\right) \text { or } x_{2}=y_{2} \neq 0 \text {. }
$$

Let us now consider another function, $\bar{\rho}: M / \equiv \times M / \equiv \rightarrow \mathrm{R}_{+}$, induced by a given $\rho$ :

$$
\bar{\rho}([x],[y]):=\inf \left\{\rho\left(x^{\prime}, y^{\prime}\right) \mid x^{\prime} \equiv x, y^{\prime} \equiv y\right\} .
$$

Remark 2.4 Evidently,

(a) even if $\rho$ is a metric, $\bar{\rho}$ need not satisfy the triangle inequality;

(b) $\bar{\rho}([x],[y])>0$ does not imply $\hat{\rho}([x],[y])>0$.

The following Lemma will prove to be very useful.

Lemma 2.5 If $\bar{\rho}$ satisfies the triangle inequality, then

$$
\hat{\rho}=\bar{\rho}
$$

Proof Evidently, for any $[x],[y] \in M / \equiv$,

$$
\hat{\rho}([x],[y]) \leq \bar{\rho}([x],[y]) .
$$

To prove the inverse inequality

$$
\hat{\rho}([x],[y]) \geq \bar{\rho}([x],[y]),
$$

let us consider the sequence of auxiliary functions $\hat{\rho}_{m}: M / \equiv \times M / \equiv \rightarrow \mathrm{R}_{+}$for $m \in \mathrm{N}$, defined by

$$
\begin{aligned}
\hat{\rho}_{m}([x],[y]):=\inf \left\{\sum_{i=1}^{m} \rho\left(p_{i}, q_{i}\right) \mid p_{1} \equiv x,\right. & \\
& \left.q_{i} \equiv p_{i+1}, \text { for } i=1, \ldots, m-1, \text { and } q_{m} \equiv y\right\} .
\end{aligned}
$$

Since $\hat{\rho}[x],[y])=\inf _{m} \hat{\rho}_{m}([x],[y])$, it suffices to prove

$$
\hat{\rho}_{m} \geq \bar{\rho}
$$

for every $m$. 
For $m=1$, we obtain equality. For $m=2$, the inequality $(2.3)_{m}$ is the triangle inequality for $\bar{\rho}$, which is satisfied by the assumption.

Assume $(2.3)_{m-1}$. Then, using arbitrary sequences $\left(p_{i}\right)_{i=1, \ldots, m-1},\left(q_{i}\right)_{i=1, \ldots, m-1}$ satisfying the suitable conditions, we obtain

$$
\bar{\rho}([x],[y]) \leq \hat{\rho}_{m-1}([x],[y]),
$$

which, together with the triangle inequality ( $m=2$ above), implies $(2.3)_{m}$.

The following will be needed in Sect. 6 .

Lemma 2.6 For any $M \neq \emptyset$, an equivalence relation $\equiv$ in $M$, and arbitrary functions $\rho_{1}, \rho_{2}: M \times M \rightarrow \mathrm{R}_{+}$, if $\rho_{1}, \rho_{2}$ are metrically equivalent, i.e. there exist $\alpha, \beta>0$ such that

$$
\beta \rho_{2} \leq \rho_{1} \leq \alpha \rho_{2}
$$

then the corresponding quotient functions $\hat{\rho}_{1}, \hat{\rho}_{2}$ are metrically equivalent as well.

Proof By the assumption, there exist $\alpha, \beta>0$ such that for every $x, y \in M$

$$
\beta \rho_{2}(x, y) \leq \rho_{1}(x, y) \leq \alpha \rho_{2}(x, y) .
$$

Thus, for every $m \geq 2, i \in\{1, \ldots, m\}$, and for all choices of $x_{i}, y_{i} \in M$ (see Definition 2.1),

$$
\beta \sum_{i=1}^{m} \rho_{2}\left(x_{i}, y_{i}\right) \leq \sum_{i=1}^{m} \rho_{1}\left(x_{i}, y_{i}\right) \leq \alpha \sum_{i=1}^{m} \rho_{2}\left(x_{i}, y_{i}\right),
$$

which implies

$$
\beta \hat{\rho}_{2}([x],[y]) \leq \hat{\rho}_{1}([x],[y]) \leq \alpha \hat{\rho}_{2}([x],[y]) .
$$

\section{Equivalence relations associated with some semigroups}

Let $\mathcal{F}$ be a semigroup of transformations of a nonempty set $M$ into itself.

If $\mathcal{F}$ is a group, then the natural equivalence $\equiv \mathcal{F}$ is defined by

$$
x \equiv \mathcal{F} y: \Longleftrightarrow \exists f \in \mathcal{F} \quad f(x)=y \text {. }
$$

For an arbitrary semigroup $\mathcal{F}$, the choice of a "natural" equivalence depends on properties of $\mathcal{F}$. We shall assume that id $\in \mathcal{F}$.

Let us consider two relations, $\stackrel{i}{=} \mathcal{F}$ for $i=1,2$ : 
Definition 3.1 For any $x, y \in M$

(a)

$$
x \stackrel{(1)}{=} \mathcal{F} y: \Longleftrightarrow \exists f, g \in \mathcal{F} f(x)=g(y)
$$

(b)

$$
x \stackrel{(2)}{=} \mathcal{F} y: \Longleftrightarrow \exists u \in M \exists f, g \in \mathcal{F}(x=f(u) \text { and } \quad y=g(u))
$$

We are looking for conditions on $\mathcal{F}$ under which these two relations are equivalences. Therefore, we extend the well-known notion of divisibility of a semigroup (Liapin 1974).

Definition 3.2 (i) $\mathcal{F}$ is weakly left divisible if and only if

$$
\forall f, g \in \mathcal{F} \exists p \in \mathcal{F} f=p g \text { or } g=p f \text {. }
$$

(ii) $\mathcal{F}$ is weakly right divisible if and only if

$$
\forall f, g \in \mathcal{F} \exists p \in \mathcal{F} f=g p \text { or } g=f p .
$$

(iii) $\mathcal{F}$ is weakly divisible whenever it is both left and right weakly divisible.

As will be shown in Example 3.6 (a) and (b), weak left divisibility does not imply weak right divisibility and conversely.

Theorem 3.3 (i) If $\mathcal{F}$ is weakly left divisible, then $\stackrel{(1)}{=} \mathcal{F}$ is an equivalence relation.

(ii) If $\mathcal{F}$ is weakly right divisible and consists of injections, then $\stackrel{(2)}{=} \mathcal{F}$ is an equivalence relation.

Proof Evidently (by Definition 3.1), both relations are reflexive and symmetric. It remains to prove their transitivity.

(i) Let $x \stackrel{(1)}{=} \mathcal{F} y$ and $y \stackrel{(1)}{=} \mathcal{F} z$; then $f(x)=g(y)$ for some $f, g \in \mathcal{F}$ and $g^{\prime}(y)=h(z)$ for some $g^{\prime}, h \in \mathcal{F}$.

Since $\mathcal{F}$ is weakly left divisible, for $g, g^{\prime}$ there is $p \in \mathcal{F}$ such that $g=p g^{\prime}$ or $g^{\prime}=p g$. Without any loss of generality, we may assume that $g=p g^{\prime}$. Then $f(x)=p g^{\prime}(y)=p h(z)$, whence $x \stackrel{(1)}{=} \mathcal{F} z$.

(ii) Let $x \stackrel{(2)}{=} \mathcal{F} y$ and $y \stackrel{(2)}{=} \mathcal{F} z$; then there exist $u, v \in M$ and $f, g, g^{\prime}, h \in \mathcal{F}$ such that $f(u)=x, g(u)=y$ and $g^{\prime}(v)=y, h(v)=z$.

Since $\mathcal{F}$ is weakly right divisible, for $g, g^{\prime}$ there is $p \in \mathcal{F}$ such that $g=g^{\prime} p$ or $g^{\prime}=g p$. As before, we may assume that $g=g^{\prime} p$.

Then $g^{\prime} p(u)=g^{\prime}(v)$, whence $p(u)=v$ because $g^{\prime}$ is injective; thus $x=f(u)$ and $z=h p(u)$, that is, $x \stackrel{(2)}{=} \mathcal{F} z$. 
Let us consider the following additional relation $\stackrel{(3)}{=} \mathcal{F}$ in $M$ :

$$
x \stackrel{(3)}{=} \mathcal{F} y: \Longleftrightarrow \exists p \in \mathcal{F} p(x)=y \text { or } p(y)=x .
$$

Proposition 3.4 (i) For every $x, y \in M$ and $j=1,2$

$$
x \stackrel{(3)}{=} \mathcal{F} y \Longrightarrow x \stackrel{(j)}{=} F y \text {. }
$$

(ii) If $\mathcal{F}$ is weakly left divisible, then for every $x, y \in M$

$$
x \stackrel{(2)}{=} \mathcal{F} y \Longleftrightarrow x \stackrel{(3)}{=} \mathcal{F} y \text {. }
$$

(iii) If $\mathcal{F}$ is weakly right divisible and consists of injections, then for every $x, y \in M$

$$
x \stackrel{(1)}{\equiv} \mathcal{F} y \Longleftrightarrow x \stackrel{(3)}{\equiv} \mathcal{F} y \text {. }
$$

Proof It is easy to see that (i) holds independently of the properties of a semigroup $\mathcal{F}$. It remains to prove the converse implications in (ii) and (iii).

(ii) Let $\mathcal{F}$ be weakly left divisible and $x \stackrel{(2)}{\equiv} \mathcal{F} y$. Then for some $u \in M$ and $f, g \in \mathcal{F}$

$$
x=f(u) \text { and } y=g(u),
$$

and there exists $p \in \mathcal{F}$ such that $f=p g$ or $g=p f$. If $f=p g$, then $x=p(y)$; if $g=p f$, then $y=p(x)$.

(iii) Let $\mathcal{F}$ be weakly right divisible and consist of injections, and let $x \stackrel{(1)}{=} \mathcal{F} y$. Then for some $f, g \in \mathcal{F}$

$$
f(x)=g(y),
$$

and there exists $p \in \mathcal{F}$ such that $f=g p$ or $g=f p$. If $f=g p$, then $g p(x)=g(y)$, whence $p(x)=y$ because $g$ is injective; if $g=f p$, then $f p(y)=f(x)$, whence $p(y)=x$, because $f$ is injective.

As a direct consequence of Proposition 3.4, we obtain the following.

Corollary 3.5 If $\mathcal{F}$ is weakly divisible and consists of injections, then the equivalence relations $\stackrel{(j)}{=} \mathcal{F}$ for $j=1,2$ coincide with the third one, $\stackrel{(3)}{=} \mathcal{F}$.

If all three relations $\stackrel{(i)}{=} \mathcal{F}$ for $i=1,2,3$ coincide, we shall briefly denote them by $\equiv \mathcal{F}$.

Example 3.6 (a) Let $A \subset M$, $\operatorname{card} A \geq 2$, and let $f_{a}(x):=a \in A$ for every $x \in M$. Then the semigroup $\mathcal{F}:=\left\{\mathrm{id}, f_{a} \mid a \in A\right\}$ is weakly left divisible but is not weakly right divisible. 
(b) Let $A \subset M$ and let $\mathcal{F}$ consist of idempotent functions $f_{i}, i=1, \ldots, k$ with $f_{i}(M)=A$, for some $k \geq 2$. Then $\mathcal{F}$ is weakly right divisible but is not weakly left divisible.

Note that the semigroup described in Example 3.6 (a) is a particular left zero semigroup, while the semigroup described in Example 3.6 (b) is a particular right zero semigroup (see Liapin 1974).

Remark 3.7 Let us observe that if $\mathcal{F}$ is a finite semigroup of injections, then $\mathcal{F}$ is a group. Indeed, let $m \geq 2$ and $\mathcal{F}=\left\{f_{1}, \ldots, f_{m}\right\}$; since each $f_{i}$ is injective, it follows that $f_{i}^{k}=f_{i}^{l}$ for some $k<l \leq m$ implies $f_{i}^{l-k}=\mathrm{id}$.

Further, for every $i$ there is an $n_{i}$ such that $f_{i}^{n_{i}}=\mathrm{id}$, because otherwise, for some $i \leq m$ and $f:=f_{i} \neq \mathrm{id}$

$$
f^{k} \neq \mathrm{id} \text { for every } k \text {. }
$$

Then, $f^{k}=f_{i_{k}}$ for a sequence $\left(i_{k}\right)$ of indices with $i_{k} \notin\left\{1, \ldots, i_{k-1}\right\}$, contrary to the assumption that $\mathcal{F}$ is finite.

Hence, evidently, for $i=1, \ldots, m$, the function $f_{i}^{n_{i}-1}$ is inverse to $f_{i}$.

Given any of the equivalence relations defined above (see Definition 3.1), we shall refer to its equivalence classes as generalized orbits or simply orbits.

Definition 3.8 Let $[x]$ be the orbit of $x \in M$ with respect to $\stackrel{(i)}{=} \mathcal{F}$ for some $i$ and let $u \in[x]$. Then,

(i) $u$ is an origin of $[x]$ if and only if for every $x^{\prime} \in[x]$ there is an $f \in \mathcal{F}$ with $f(u)=x^{\prime}$;

(ii) $u$ is an end of $[x]$ if and only if for every $x^{\prime} \in[x]$ there is an $f \in \mathcal{F}$ with $f\left(x^{\prime}\right)=u$.

Remark 3.9 Of course, for some semigroups neither origin nor end of an orbit exists; for instance, this happens for $\mathcal{F}$ that consists of all translations of $\mathrm{R}^{n}$ about $t v$ for a given vector $v \neq 0$ and arbitrary nonnegative $t$.

On the other hand, it may happen that every member of an orbit is its origin as well as its end.

\section{Quotient semi-metric spaces of generalized orbits}

We are now going to give various geometric examples. They concern isometric embeddings of the space $\left(\mathcal{K}^{n}, \rho_{H}\right)$ into itself (Example 4.1) and weak contractions of $\left(\mathcal{K}_{0}^{n}, \delta\right)$ and of Euclidean $n$-space (Example 4.3 and Examples 4.4, 4.5, respectively).

As is well known, every isometric embedding of Euclidean space is an isometry. For the space $\left(\mathcal{K}^{n}, \rho_{H}\right)$ the situation is different: any Minkowski translation $\phi: \mathcal{K}^{n} \rightarrow \mathcal{K}^{n}$ (defined by $\phi(X):=X+A$ for some $A \in \mathcal{K}^{n}$ ) is an isometric embedding, but is not surjective if $A$ is not a singleton. It was proved by Schneider (1975) that an isometric embedding of $\left(\mathcal{K}^{n}, \rho_{H}\right)$ into itself is an isometry if and only if it is induced by an Euclidean isometry. As was proved by Gruber and Lettl (1980), a map $f: \mathcal{K}^{n} \rightarrow \mathcal{K}^{n}$ is an 
isometric embedding with respect to $\rho_{H}$ whenever it is of the form $\phi g^{*}$ for some Euclidean isometry $g$ and Minkowski translation $\phi$ (see also Schneider 1993, Note 9, p. 59).

Example 4.1 (a) Let $M:=\mathcal{K}^{n}, \rho:=\rho_{H}$ and let $A \in \mathcal{K}^{n}$. For every $\alpha \geq 0$ let $f_{\alpha}$ be the Minkowski translation about $\alpha A$ :

$$
f_{\alpha}(X):=X+\alpha A \text {. }
$$

Then $\mathcal{F}_{A}:=\left\{f_{\alpha} \mid \alpha \geq 0\right\}$ is a weakly divisible semigroup of isometric embeddings and thus, by Corollary 3.5, all three relations $\stackrel{(i)}{=} \mathcal{F}_{A}$ for $i=1,2,3$ coincide. As will be proved later (see Lemma 5.6), if $\operatorname{dim} A \geq 1$, then for every $X \in \mathcal{K}^{n}$ the orbit $[X]_{\equiv \mathcal{F}_{A}}$ has a unique origin.

(b) Let $A \in \mathcal{K}^{n}$ and let $\mathcal{F}_{A}$ be as in (a). Assume $G$ to be a subgroup of $O(n)$ with all elements being Euclidean self-isometries of $A$.

For any $g \in G$, let $g^{\star}: \mathcal{K}^{n} \rightarrow \mathcal{K}^{n}$ be the map induced by $g$, i.e. $g^{\star}(X):=g(X)$, the image of $X$ under $g$. If

$$
\mathcal{G}:=\left\{g^{\star} \mid g \in G\right\}
$$

then $\mathcal{G} \circ \mathcal{F}_{A}$ is a weakly divisible semigroup of isometric embeddings.

Moreover, by the assumption on $G$,

$$
g^{\star} \circ f_{\alpha}=f_{\alpha} \circ g^{\star} \text { for every } g \in G \text { and } f_{\alpha} \in \mathcal{F}_{A} \text {. }
$$

Remark 4.2 The semigroup $\mathcal{F}$ of all Minkowski translations of $\mathcal{K}^{n}$ is neither weakly left nor weakly right divisible. Indeed, by commutativity of $\mathcal{F}$, it suffices to prove that $\mathcal{F}$ is not weakly left divisible.

Let $A$ be an n-simplex, $B=B^{n}$, and consider $f, g \in \mathcal{F}$ :

$$
f(x):=X+A, g(X):=X+B \text { for every } X \in \mathcal{K}^{n} .
$$

Suppose $p f=g$ or $p g=f$ for some $p \in \mathcal{F}$. Then $p(X)=X+C$ for some $C \in \mathcal{K}^{n}$ and either $X+A+C=X+B$ or $X+B+C=X+A$ for every $X$. Thus, $A+C=B$ or $B+C=A$, which is impossible, because neither a ball is a summand of a simplex (see Schneider 1993, Cor. 3.2.13) nor a simplex is a summand of a ball (see Schneider 1993, Th. 3.2.8).

Example 4.3 Let $M:=\mathcal{K}_{0}^{n}$. Consider a hyperplane $H$ in $\mathrm{R}^{n}$ and let $S_{H}: \mathcal{K}_{0}^{n} \rightarrow$ $\mathcal{K}_{0}^{n}$ be Steiner symmetrization (see Moszyńska 2006, p. 41). Then the semigroup $\mathcal{F}:=\left\{\mathrm{id}, S_{H}\right\}$ is weakly divisible.

Since, by definition of Steiner symmetrization, for every $X \in \mathcal{K}^{n}$ the set $S_{H}(X)$ is symmetric with respect to $H$, it follows that $S_{H} S_{H}=S_{H}$. Thus the generalized orbit of $X$ with respect to $\stackrel{(1)}{=}_{F}$ is the inverse image $S_{H}^{-1}\left(S_{H}(X)\right)$ of $S_{H}(X)$. Moreover, $S_{H}(X)$ is the unique end of $[X]$. 
Let us notice that $S_{H}$ is a weak contraction with respect to the metric $\delta$ of symmetric difference: for any convex bodies $X, Y$,

$$
\delta\left(S_{H}(X), S_{H}(Y)\right) \leq \delta(X, Y)
$$

(compare Volčič 2011, where this inequality has been proved for a larger collection of sets; our proof is simpler). Indeed, since $S_{H}(X \cap Y) \subset S_{H}(X) \cap S_{H}(Y)$, it follows that

$$
V_{n}\left(S_{H}(X \cap Y)\right) \leq V_{n}\left(S_{H}(X) \cap S_{H}(Y)\right)
$$

As is well known, the Steiner symmetrization preserves volume $V_{n}$ (see Moszyńska 2006). Hence, by (4.2), it is easy to calculate that

$$
\begin{aligned}
\delta\left(S_{H}(X), S_{H}(Y)\right) & =V_{n}\left(S_{H}(X)\right)+V_{n}\left(S_{H}(Y)\right)-2 V_{n}\left(S_{H}(X) \cap S_{H}(Y)\right) \\
& \leq V_{n}(X)+V_{n}(Y)-2 V_{n}(X \cap Y)=\delta(X, Y) .
\end{aligned}
$$

Example 4.4 Let now $M:=\mathrm{R}^{n}$ and $A \in \mathcal{K}^{n}$. Consider the metric projection $\xi_{A}$ : $\mathrm{R}^{n} \rightarrow \mathrm{R}^{n}$, which assigns to any $x \in \mathrm{R}^{n}$ the unique point of $A$ nearest to $x$. Again, the semigroup $\mathcal{F}:=\left(\mathrm{id}, \xi_{A}\right)$ is weakly divisible.

As is well known, $\xi_{A}$ is a weak contraction with respect to the Euclidean metric (see Moszyńska 2006). This map is a retraction too, and the orbit $[x]$ with respect to $\stackrel{[1]}{=} \mathcal{F}$ is the inverse image of $\xi_{A}(x)$. Moreover, $\xi_{A}(x)$ is the unique end of $[x]$.

Example 4.5 Let now $\mathcal{F}^{A}$ consist of id and the metric projections of $\mathrm{R}^{n}$ onto the parallel bodies of a given $A \in \mathcal{K}^{n}$ :

$$
\mathcal{F}^{A}:=\left\{\mathrm{id}, \xi_{A+t B^{n}} \mid t \geq 0\right\}
$$

Then $\mathcal{F}^{A}$ is weakly divisible. It consists of weak contractions.

The generalized orbit of an $x$ with respect to $\stackrel{(1)}{=} \mathcal{F}^{A}$ is the union of inverse images:

$$
[x]=\bigcup_{f, g \in \mathcal{F}^{A}} f^{-1}(g(x)) .
$$

The element $\xi_{A}(x)$ is the unique end of $[x]$.

Let us now return to the quotient semi-metric $\hat{\rho}$ (determined by a metric $\rho$ in $M$ ) in a quotient set of $M$ (see Definition 2.1 and Proposition 2.2), and to the function $\bar{\rho}$ defined by formula (2.1). Since the definition of the second one, $\bar{\rho}$, is much simpler than that of the first, $\hat{\rho}$, it is important to know when these two functions coincide. This information will be useful in Sect. 5, where we deal with the problem under what assumptions on $(M, \rho)$ the quotient semi-metric $\hat{\rho}$ is a metric.

The next three Theorems 4.6-4.8 generalize Lemma 3.3.6 in Burago et al. (2001).

First, we make use of Theorem 3.3(i). 
Theorem 4.6 Let $\mathcal{F}$ be a semigroup of weak contractions of a metric space $(M, \rho)$ into itself. If $\mathcal{F}$ is weakly left divisible, then for $\stackrel{(1)}{=} \mathcal{F}$ the corresponding functions $\hat{\rho}$ and $\bar{\rho}$ coincide.

Proof By Theorem 3.3.(i), $\stackrel{(1)}{=} \mathcal{F}$ is an equivalence relation. In view of Lemma 2.5, it suffices to show that $\bar{\rho}$ satisfies the triangle inequality.

Let us consider the orbits $\left[x_{0}\right],\left[y_{0}\right],\left[z_{0}\right]$ of three points in $M$, with respect to $\stackrel{(1)}{=} \mathcal{F}$. Let $x \in\left[x_{0}\right], z, z^{\prime} \in\left[z_{0}\right]$, and $y \in\left[y_{0}\right]$. Then, by Definition 3.1 (a), there exist $f, f^{\prime} \in \mathcal{F}$ and $u \in M$ such that $f(z)=f^{\prime}\left(z^{\prime}\right)=u$. Thus,

$$
\begin{aligned}
\rho(x, z)+\rho\left(z^{\prime}, y\right) & \geq \rho(f(x), f(z))+\rho\left(f^{\prime}\left(z^{\prime}\right), f^{\prime}(y)\right) \\
& =\rho(f(x), u)+\rho\left(u, f^{\prime}(y)\right) \geq \rho\left(f(x), f^{\prime}(y)\right),
\end{aligned}
$$

whence, by Proposition 3.4(i),

$$
\rho(x, z)+\rho\left(z^{\prime}, y\right) \geq \bar{\rho}\left(\left[x_{0}\right],\left[y_{0}\right]\right) .
$$

Passing to the infimum on the left side, we obtain the required triangle inequality for $\bar{\rho}$.

Next, we make use of Theorem 3.3(ii):

Theorem 4.7 Let $\mathcal{F}$ be a semigroup of weakly expanding bijections of a metric space $(M, \rho)$ onto itself. If $\mathcal{F}$ is weakly right divisible, then for the equivalence relation $\stackrel{(2)}{=} \mathcal{F}$ the corresponding functions $\hat{\rho}$ and $\bar{\rho}$ coincide.

Proof As above, in view of Lemma 2.5 it suffices to prove the triangle inequality for $\bar{\rho}$.

Consider the orbits $\left[x_{0}\right],\left[y_{0}\right],\left[z_{0}\right]$ of three points in $M$, with respect to $\stackrel{(2)}{=} \mathcal{F}$. Let $x \in\left[x_{0}\right], z, z^{\prime} \in\left[z_{0}\right]$, and $y \in\left[y_{0}\right]$. Then, by Definition 3.1 (b), there exist $f, f^{\prime} \in \mathcal{F}$ and $u \in M$ such that $f(u)=z$ and $f^{\prime}(u)=z^{\prime}$. Since $f$ and $f^{\prime}$ are surjections, it follows that $x=f(\tilde{x})$ and $y=f^{\prime}(\tilde{y})$ for some $\tilde{x} \in\left[x_{0}\right]$ and $\tilde{y} \in\left[y_{0}\right]$. Thus,

$$
\begin{aligned}
\rho(x, z)+\rho\left(z^{\prime}, y\right) & =\rho(f(\tilde{x}), f(u))+\rho\left(f^{\prime}(u), f^{\prime}(\tilde{y})\right) \\
& \geq \rho(\tilde{x}, u)+\rho(u, \tilde{y}) \geq \rho(\tilde{x}, \tilde{y}) \geq \bar{\rho}\left(\left[x_{0}\right],\left[y_{0}\right]\right) .
\end{aligned}
$$

Hence

$$
\rho(x, z)+\rho\left(z^{\prime}, y\right) \geq \bar{\rho}\left(\left[x_{0}\right],\left[y_{0}\right]\right) .
$$

Passing to the infimum on the left side, we obtain the triangle inequality for $\bar{\rho}$.

Finally, let us prove the following.

Theorem 4.8 If $\mathcal{F}$ is a weakly divisible semigroup of injections of a metric space $(M, \rho)$ into itself and every $f \in \mathcal{F}$ is either a weak contraction or a surjective weak expansion, then for all three equivalence relations $\stackrel{(i)}{=} \mathcal{F}, i=1,2,3$, the corresponding functions $\hat{\rho}$ and $\bar{\rho}$ coincide. 
Proof By Corollary 3.5, all three relations $\stackrel{(i)}{=}_{\mathcal{F}}$ for $i=1,2,3$ coincide.

By Lemma 2.5, it suffices to prove the triangle inequality for the corresponding function $\bar{\rho}$.

Consider the orbits $\left[x_{0}\right],\left[y_{0}\right],\left[z_{0}\right]$ of three points in $M$ with respect to $\equiv \mathcal{F}$. Let $x \in\left[x_{0}\right], z, z^{\prime} \in\left[z_{0}\right], y \in\left[y_{0}\right]$. There exists $p \in \mathcal{F}$ such that $z^{\prime}=p(z)$ or $z=p\left(z^{\prime}\right)$. We may assume that $z^{\prime}=p(z)$.

Let $p$ be a weak contraction. Then

$$
\begin{aligned}
& \rho(x, z)+\rho\left(z^{\prime}, y\right)=\rho(x, z)+\rho(p(z), y) \geq \\
& \quad \rho(p(x), p(z))+\rho(p(z), y) \geq \rho(p(x), y) \geq \bar{\rho}\left(\left[x_{0}\right],\left[y_{0}\right]\right) .
\end{aligned}
$$

Passing to the infimum on the left side, we obtain the triangle inequality for $\bar{\rho}$.

Let now $p$ be a weak expansion. Then, by the surjectivity of $p$, there exists an $\tilde{y} \in M$ such that $y=p(\tilde{y})$. Hence,

$$
\begin{aligned}
\rho(x, z)+\rho\left(z^{\prime}, y\right) & =\rho(x, z)+\rho(p(z), y) \\
& =\rho(x, z)+\rho(p(z), p(\tilde{y})) \geq \rho(x, z)+\rho(z, \tilde{y}) \geq \rho(x, \tilde{y}) .
\end{aligned}
$$

But $\tilde{y} \in\left[y_{0}\right]$; thus,

$$
\rho(x, z)+\rho\left(z^{\prime}, y\right) \geq \bar{\rho}\left(\left[x_{0}\right],\left[y_{0}\right]\right) .
$$

Passing to the infimum on the left side, we obtain the triangle inequality for $\bar{\rho}$.

Remark 4.9 The following example shows that in Theorem 4.8 the assumption concerning weak contractions and weak expansions is essential.

Let $M=\left\{x, x^{\prime}, y, y^{\prime}, z\right\} \subset \mathrm{R}$,

$$
x=4, x^{\prime}=6, y=2, y^{\prime}=3, z=0,
$$

and let $\mathcal{F}:=\{f$, id $\}$, where $f: M \rightarrow M$ is defined by

$$
f(x)=x^{\prime}, f(y)=y^{\prime}, f\left(x^{\prime}\right)=x, f\left(y^{\prime}\right)=y, f(z)=z .
$$

Then $\mathcal{F}$ is a divisible semigroup of bijections, but $f \mid\{x, y, z\}$ is an expansion and $f \mid\left\{x^{\prime}, y^{\prime}, z\right\}$ is a contraction with respect to the Euclidean metric $\rho \mid M \times M$.

Since $\rho(x, y)=2, \rho\left(x, y^{\prime}\right)=1, \rho(x, z)=4, \rho(y, z)=2, \rho\left(x^{\prime}, y\right)=4$, $\rho\left(x^{\prime}, y^{\prime}\right)=3, \rho\left(x^{\prime}, z\right)=6, \rho\left(y^{\prime}, z\right)=3$, and $[x]=\left\{x, x^{\prime}\right\},[y]=\left\{y, y^{\prime}\right\},[z]=\{z\}$, it follows that

$$
\bar{\rho}([x],[y])+\bar{\rho}([y],[z])=1+2=3<4=\bar{\rho}([x],[z])
$$

and thus $\bar{\rho} \neq \hat{\rho}$ in view of Proposition 2.2. 


\section{Quotient metric spaces of generalized orbits}

In Sect. 4 we were concerned with quotient semi-metric spaces of (generalized) orbits w.r.t. some semigroups of transformations. Now, the question is when such a semimetric is a metric.

By Corollary 3.5, if $\mathcal{F}$ is a weakly divisible semigroup of injections of a metric space $(M, \rho)$ into itself, then the three equivalence relations coincide. In such case we simply use the symbol $\equiv \mathcal{F}$ for all of them.

Let us first prove the following.

Theorem 5.1 If $\mathcal{F}$ is a weakly divisible semigroup of isometric embeddings of a metric space $(M, \rho)$ into itself and the orbits w.r.t. $\equiv \mathcal{F}$ are closed in $(M, \rho)$, then $\hat{\rho}$ is a metric.

Proof By Theorem 4.6, for $\equiv_{\mathcal{F}}$, since all elements of $\mathcal{F}$ are weak contractions, it follows that $\hat{\rho}=\bar{\rho}$.

Thus, it remains to prove that $\bar{\rho}$ is non-degenerate. Assume $\bar{\rho}([x],[y])=0$ for some $x, y \in M$. Then, there exist sequences $\left(x_{k}\right)_{k \in \mathrm{N}}$ in $[x]$ and $\left(y_{k}\right)_{k \in \mathrm{N}}$ in $[y]$ such that

$$
\lim _{k \rightarrow \infty} \rho\left(x_{k}, y_{k}\right)=0
$$

Further, there exist sequences $\left(f_{k}\right)_{k \in \mathrm{N}}$ and $\left(g_{k}\right)_{k \in \mathrm{N}}$ in $\mathcal{F}$ such that for all $k$ either

$$
f_{k}\left(x_{k}\right)=x
$$

or

$$
x_{k}=f_{k}(x)
$$

and, similarly, either

$$
g_{k}\left(y_{k}\right)=y
$$

or

$$
y_{k}=g_{k}(y) .
$$

Hence, there is a subsequence $\left(j_{k}\right)_{k \in \mathrm{N}}$ satisfying (5.2) and (5.4), or (5.2) and (5.5), or (5.3) and (5.4), or (5.3) and (5.5) for all $j_{k}$.

If (5.2) holds for all $j_{k}$, then $\rho\left(x_{j_{k}}, y_{j_{k}}\right)=\rho\left(f_{j_{k}}\left(x_{j_{k}}\right), f_{j_{k}}\left(y_{j_{k}}\right)\right)=\rho\left(x, f_{j_{k}}\left(y_{j_{k}}\right)\right)$, whence, by (5.1), $x=\lim f_{j_{k}}\left(y_{j_{k}}\right)$, and thus $x \in[y]$, because [y] is closed. Therefore $[x]=[y]$.

If (5.4) holds for all $j_{k}$, then the reasoning is analogous.

Thus, it remains to assume (5.3) and (5.5). 
Since $\mathcal{F}$ is weakly right divisible, we may assume (passing to a subsequence if needed) that for $f_{j_{k}}$ and $g_{j_{k}}$ there exists a $p_{j_{k}}$ such that $f_{j_{k}} p_{j_{k}}=g_{j_{k}}$. Then

$$
\rho\left(x_{j_{k}}, y_{j_{k}}\right)=\rho\left(f_{j_{k}}(x), g_{j_{k}}(y)\right)=\rho\left(f_{j_{k}}(x), f_{j_{k}} p_{j_{k}}(y)\right)=\rho\left(x, p_{j_{k}}(y)\right)
$$

Hence, by (5.1), $x=\lim p_{j_{k}}(y)$ and thus $x \in[y]$, because $[y]$ is closed. Therefore $[x]=[y]$, which completes the proof.

It is easy to see that even if $\mathcal{F}$ is a group of isometries of Euclidean space $\left(\mathrm{R}^{n}, \rho\right)$, orbits need not be closed, whence $\hat{\rho}$ need not be a metric (compare Remark 2.3).

Corollary 5.2 Let $G$ be a group of isometries of Euclidean space $\left(\mathrm{R}^{n}, \rho\right)$ and let $\mathcal{G}=\left\{f^{*} \mid f \in G\right\}$, where $f^{*}$ is the isometry of $\left(\mathcal{K}^{n}, \rho_{H}\right)$ induced by $f$. If $G$ is closed in the group $\operatorname{Iso}(n)$ (of all isometries of $\mathrm{R}^{n}$ ) in topology of uniform convergence, then $\hat{\rho_{H}}$ is a metric in $\mathcal{K}^{n} / \equiv \mathcal{G}$.

Proof In view of Theorem 5.1, it suffices to show that orbits w.r.t. $\mathcal{G}$ are closed. The proof of this fact is the same as that of Proposition 3.5 in Herburt and Moszyńska (2009), that concerns the group Iso( $n)$.

We now pass to weak contractions and weak expansions.

Theorem 5.3 If $\mathcal{F}$ is a weakly left divisible semigroup of weak contractions of a metric space $(M, \rho)$ into itself, the orbits w.r.t. $\stackrel{(1)}{=} \mathcal{F}$ are closed in $(M, \rho)$ and every orbit has at least one end, then

(a) For every two orbits $[x],[y]$,

$$
\hat{\rho}([x],[y])=\inf \{\rho(u, v) \mid \mathrm{u} \text { is an end of }[x] \text { and } v \text { is an end of }[y]\} \text {; }
$$

(b) $\hat{\rho}$ is a metric.

Proof In view of Theorem 4.6, $\hat{\rho}=\bar{\rho}$.

(a) Take $x, y \in M$ and let $u$ and $v$ be ends of $[x]$ and $[y]$, respectively.

Then, for every $x^{\prime} \in[x]$ and $y^{\prime} \in[y]$ there exist $f, g \in \mathcal{F}$ such that $u=f\left(x^{\prime}\right)$ and $v=g\left(y^{\prime}\right)$, whence

$$
\rho\left(x^{\prime}, y^{\prime}\right) \geq \rho\left(f\left(x^{\prime}\right), f\left(y^{\prime}\right)\right)=\rho\left(u, f\left(y^{\prime}\right)\right)
$$

and

$$
\rho\left(x^{\prime}, y^{\prime}\right) \geq \rho\left(g\left(x^{\prime}\right), g\left(y^{\prime}\right)\right)=\rho\left(g\left(x^{\prime}\right), v\right) \text {. }
$$

Further, by the weak left divisibility of $\mathcal{F}$, there exists $p \in \mathcal{F}$ such that

$$
f=p g \text { or } g=p f \text {. }
$$


In the first case $f\left(y^{\prime}\right)=p(v)$, whence, by (5.6), $\rho\left(x^{\prime}, y^{\prime}\right) \geq \rho(u, p(v))$ and thus

$$
\bar{\rho}(x, y) \geq \inf _{p \in \mathcal{F}} \rho(u, p(v))
$$

where $p(v)$ is again an end of $[y]$.

In the second case the reasoning is analogous.

By properties of infimum, we obtain the converse inequality. Hence (a) is satisfied.

(b) In view of Theorem 4.6, it suffices to prove that $\bar{\rho}$ is non-degenerate. Let $\bar{\rho}([x],[y])=0$ for some $x, y \in M$. Since both orbits are closed, from (a) it follows that $[x]=[y]$.

Theorem 5.4 If $\mathcal{F}$ is a weakly right divisible semigroup of weakly expanding bijections of a metric space $(M, \rho)$ onto itself, the orbits w.r.t. $\stackrel{(2)}{=} \mathcal{F}$ are closed in $(M, \rho)$ and every orbit has at least one origin, then

(a) For every two orbits $[x],[y]$,

$$
\hat{\rho}([x],[y])=\inf \{\rho(u, v) \mid u \text { is an origin of }[x] \text { and } v \text { is an origin of }[y]\} \text {; }
$$

(b) $\hat{\rho}$ is a metric.

Proof In view of Theorem 4.7, $\hat{\rho}=\bar{\rho}$.

(a) Take $x, y \in M$ and let $u$ and $v$ be origins of $[x]$ and [y], respectively. Then

$$
\bar{\rho}([x],[y])=\inf \{\rho(f(u), g(v)) \mid f, g \in \mathcal{F}\}
$$

and thus, by the weak right divisibility of $\mathcal{F}$, either

$$
\bar{\rho}([x],[y])=\inf \left\{\rho(f(u), f p(v) \mid f, p \in \mathcal{F}\} \geq \inf _{p \in \mathcal{F}} \rho(u, p(v))\right.
$$

or

$$
\bar{\rho}([x],[y])=\inf \{\rho(g p(u), g(v)) \mid g, p \in \mathcal{F}\} \geq \inf _{p \in \mathcal{F}} \rho(p(u), v) .
$$

Since every $p \in \mathcal{F}$ is surjective, in the first case $u=p\left(u^{\prime}\right)$ for some $u^{\prime} \in M$ and then $u^{\prime}$ is again an origin of $[x]$; hence

$$
\bar{\rho}([x],[y]) \geq \inf \{\rho(u, v) \mid u \text { is an origin of }[x] \text { and } v \text { is an origin of }[y]\} .
$$

In the second case the reasoning is analogous.

(b) It remains to prove that $\bar{\rho}$ is non-degenerate.

Assume $\bar{\rho}([x],[y])=0$ for some $x, y \in M$. Then, by (a), since orbits are closed, it follows that $[x]=[y]$.

The following example shows that in Theorem 5.3 the assumption on the existence of ends is essential, and similarly, in Theorem 5.4 the assumption on the existence of origins is essential. 
Example 5.5 Let $M=\mathrm{R}^{n} \backslash\{0\}$ for some $n \geq 1$.

(i) Let $\mathcal{F}$ be the semigroup of dilatations of scale $t \leq 1$. Then the orbits have no ends while all the remaining assumptions of Theorem 5.3 are satisfied. Evidently, the semi-metric $\bar{\rho}$ (and so $\hat{\rho}$ ) is degenerate.

(ii) Let $\mathcal{F}$ be the semigroup of dilatations of scale $t \geq 1$. Then the orbits have no origins while all the remaining assumptions of Theorem 5.4 are satisfied. Evidently, the semi-metric $\bar{\rho}$ (and so $\hat{\rho}$ ) is degenerate.

Let us now return to Examples 4.1 and 4.3-4.5.

Lemma 5.6 Let $A \in \mathcal{K}^{n}$. If $\operatorname{dim} A \geq 1$, then for every $X \in \mathcal{K}^{n}$ the generalized orbit of $X$ with respect to $\equiv \mathcal{F}_{A}$ has a unique origin.

Proof Take $X \in \mathcal{K}^{n}$ and let

$$
S(X):=\left\{\alpha \geq 0 \mid \exists X^{\prime} \in \mathcal{K}^{n} X=X^{\prime}+\alpha A\right\} .
$$

Then the set $S(X)$ is nonempty and bounded.

Let $s(X):=\sup S(X)$. Let us prove that $s(X) \in S(X)$. Indeed, $s(X)=\lim _{k} \alpha_{k}$ for some sequence $\left(\alpha_{k}\right)_{k \in \mathrm{N}}$ in $S(X)$. Thus, by (5.8), there is a sequence $\left(X_{k}^{\prime}\right)_{k \in N}$ in $\mathcal{K}^{n}$ such that $X=X_{k}^{\prime}+\alpha_{k} A$ for every $k$. This sequence is bounded because $\left(\alpha_{k}\right)_{k \in \mathrm{N}}$ is. Since $\mathcal{K}^{n}$ is finitely compact (see Moszyńska 2006, pp. 7-9), $\left(X_{k}^{\prime}\right)_{k \in \mathrm{N}}$ has a convergent subsequence, and so it may be assumed to be convergent to an $X_{0} \in \mathcal{K}^{n}$. Thus $X=X_{0}+s(X) A$.

It remains to prove that $X_{0}$ is a unique origin of $[X]_{\mathcal{F}_{A}}$. Let $X^{\prime} \in[X]_{\mathcal{F}_{A}}$.

If $X^{\prime}=X+\alpha A$ for some $\alpha$, then $X^{\prime}=X_{0}+(s(X)+\alpha) A$. If $X=X^{\prime}+\alpha A$ for some $\alpha$, then $\alpha \leq s(X)$ and thus $X^{\prime}=X_{0}+(s(X)-\alpha) A$.

Thus $X_{0}$ is an origin $[X]_{\mathcal{F}_{A}}$. Moreover, it is unique because it has no summand $\alpha A$ for $\alpha>0$.

Proposition 5.7 Let $A \in \mathcal{K}^{n}$ and let $\mathcal{F}_{A}$ be the semigroup of Minkowski translations $f_{\alpha}: \mathcal{K}^{n} \rightarrow \mathcal{K}^{n}$ about all $\alpha A$ for $\alpha \geq 0$. If $\operatorname{dim} A \geq 1$, then the quotient semi-metric $\hat{\rho_{H}}$ in $\mathcal{K}^{n} / \equiv \mathcal{F}_{A}$ is a metric and

$$
\hat{\rho_{H}}([X],[Y])=\rho_{H}\left(X_{0}, Y_{0}\right),
$$

where $X_{0}$ and $Y_{0}$ are the unique origins of the orbits $[X]$ and $[Y]$, respectively.

Proof In view of Theorem 5.4 and Lemma 5.6, it suffices to prove that (generalized) orbits w.r.t. $\mathcal{F}_{A}$ are closed in $\left(\mathcal{K}^{n}, \rho_{H}\right)$.

Take an $X \in \mathcal{K}^{n}$ and a sequence $\left(X_{k}\right)_{k \in \mathrm{N}}$ in the orbit $[X]$, Hausdorff convergent to an $X_{0} \in \mathcal{K}^{n}$. We are going to show that $X_{0} \in[X]$.

Since $X_{k} \equiv{ }_{F_{A}} X$ for every $k$, it follows that

$$
\forall k \exists \alpha_{k} \geq 0\left(X=X_{k}+\alpha_{k} A \text { or } X_{k}=X+\alpha_{k} A\right)
$$


Case 1 There is an infinite sequence $\left(i_{k}\right)_{k \in \mathrm{N}}$ such that

$$
X=X_{i_{k}}+\alpha_{i_{k}} A \text { for all } k \in \mathrm{N} \text {. }
$$

Then,

$$
\forall u \in S^{n-1} h(X, u)=h\left(X_{i_{k}}, u\right)+\alpha_{i_{k}} h(A, u)
$$

and thus, since $\left(X_{k}\right)$ is convergent to $X_{0} \in \mathcal{K}^{n}$, it follows that the sequence $\left(\alpha_{i_{k}}\right)_{k \in \mathrm{N}}$ is bounded; indeed, otherwise $X$ would be unbounded, contrary to its compactness. Hence the sequence $\alpha_{i_{k}}$ has a convergent subsequence and so we may assume it is convergent to an $\alpha_{0} \geq 0$. Therefore, $X=X_{0}+\alpha_{0} A$ by (4.6), whence $X_{0} \in[X]$.

Case 2 There is an infinite sequence $\left(j_{k}\right)_{k \in \mathrm{N}}$ such that

$$
X_{j_{k}}=X+\alpha_{j_{k}} A \text { for all } k \in \mathrm{N} \text {. }
$$

Again, $\alpha_{j_{k}}$ is bounded, because the sequence $\left(X_{j_{k}}\right)_{k \in \mathrm{N}}$ has compact limit; thus we may assume $\alpha_{j_{k}} \rightarrow \alpha_{0}$ for some $\alpha_{0} \geq 0$. Therefore, by (5.10), $X_{0}=X+\alpha_{0} A$, whence $X_{0} \in[X]$.

Remark 5.8 It is easy to see that in Examples 4.3-4.5 the assumptions of Theorem 5.3 are satisfied and thus the corresponding quotient semi-metrics induced are quotient metrics.

\section{Rådström space as a quotient metric space}

Let us briefly recall definition of Rådström space, called also MRH-space (MinkowskiRådström-Hörmander space); see Pallaschke and Urbański (2002).

Consider the equivalence relation $\sim$ in $\mathcal{K}^{n} \times \mathcal{K}^{n}$ :

$$
(A, B) \sim\left(A^{\prime}, B^{\prime}\right): \Longleftrightarrow A+B^{\prime}=A^{\prime}+B
$$

and let $[A, B]$ be the equivalence class of $(A, B)$ with respect to $\sim$.

In the quotient set $\left(\mathcal{K}^{n} \times \mathcal{K}^{n}\right) / \sim$ the addition and multiplication by scalars are defined by the formulae:

$$
[A, B]+[C, D]:=[A+C, B+D]
$$

and

$$
\alpha \cdot[A, B]:= \begin{cases}{[\alpha A, \alpha B]} & \text { if } \alpha \geq 0 \\ {[-\alpha B,-\alpha A]} & \text { if } \alpha \leq 0\end{cases}
$$

Then, in particular, $-[A, B]=[B, A]$. 
The structure $\left.\left(\mathcal{K}^{n} \times \mathcal{K}^{n}\right) / \sim,+, \cdot\right)$ is a linear space with $[A, A]$ being the neutral element of its additive group.

Further, the function $\|\cdot\|:\left(\mathcal{K}^{n} \times \mathcal{K}^{n}\right) / \sim \rightarrow \mathrm{R}_{+}$defined by the formula

$$
\|[A, B]\|:=\rho_{H}(A, B)
$$

is a norm. The normed linear space $\left(\left(\mathcal{K}^{n} \times \mathcal{K}^{n}\right) / \sim,+, \cdot,\|\cdot\|\right)$ is referred to as Rådström space.

We are interested in relationships between the metric $\rho_{\|\cdot\|}$ induced by the norm $\|\cdot\|$, which is defined by the formula

$$
\rho_{\|\cdot\|}([A, B],[C, D]):=\|[A, B]-[C, D]\|,
$$

and the quotient semi-metric $\hat{\rho}$ for some product metric $\rho:\left(\mathcal{K}^{n} \times \mathcal{K}^{n}\right)^{2} \rightarrow \mathrm{R}_{+}($see Herburt and Moszyńska 1991).

Theorem 6.1 If $\rho:\left(\mathcal{K}^{n} \times \mathcal{K}^{n}\right)^{2} \rightarrow \mathrm{R}_{+}$is the "taxi metric" with respect to $\rho_{H}$ in $\mathcal{K}^{n}$, that is,

$$
\rho((A, B),(C, D)):=\rho_{H}(A, C)+\rho_{H}(B, D)
$$

then $\hat{\rho}=\rho_{\|\cdot\| \cdot}$

Proof According to (2.1), let

$$
\begin{aligned}
& \bar{\rho}([A, B],[C, D]) \\
& \quad=\inf \left\{\rho\left(\left(A^{\prime}, B^{\prime}\right),\left(C^{\prime} D^{\prime}\right)\right) \mid\left(A^{\prime}, B^{\prime}\right) \sim(A, B) \text { and }\left(C^{\prime} D^{\prime}\right) \sim(C, D)\right\} .
\end{aligned}
$$

Then, by definition of $\rho$ combined with (6.1), and by the invariance of $\rho_{H}$ w.r.t. Minkowski addition, for any $\left(A^{\prime}, B^{\prime}\right) \in[A, B]$ and $\left(C^{\prime}, D^{\prime}\right) \in[C, D]$

$$
\begin{aligned}
& \rho\left(\left(A^{\prime}, B^{\prime}\right),\left(C^{\prime}, D^{\prime}\right)\right)=\rho_{H}\left(A^{\prime}, C^{\prime}\right)+\rho_{H}\left(B^{\prime}, D^{\prime}\right) \\
& \quad=\rho_{H}\left(A^{\prime}+D^{\prime}, C^{\prime}+D^{\prime}\right)+\rho_{H}\left(B^{\prime}+C^{\prime}, D^{\prime}+C^{\prime}\right) \geq \rho_{H}\left(A^{\prime}+D^{\prime}, B^{\prime}+C^{\prime}\right) \\
& \quad=\rho_{H}(A+D, B+C)=\|[A, B]-[C, D]\| .
\end{aligned}
$$

Passing to the infimum, we infer that

$$
\bar{\rho}([A, B],[C, D]) \geq\|[A, B]-[C, D]\| .
$$

Further, if $\left(A^{\prime}, B^{\prime}\right) \sim(A, B)$ and $\left(C^{\prime}, D^{\prime}\right) \sim(C, D)$, then

$$
\left(A^{\prime}, B^{\prime}\right) \sim(A+D, B+D) \text { and }\left(C^{\prime}, D^{\prime}\right) \sim(C+B, D+B)
$$


hence

$$
\begin{aligned}
\bar{\rho} & ([A, B],[C, D]) \\
& =\inf \left\{\rho\left(A^{\prime}, B^{\prime}\right),\left(C^{\prime}, D^{\prime}\right) \mid\left(A^{\prime}, B^{\prime}\right) \sim(A, B) \quad \text { and }\left(C^{\prime}, D^{\prime}\right) \sim(C, D)\right\} \\
\leq & \rho((A+D, B+D),(C+B, D+B))=\rho_{H}(A+D, C+B) \\
& +\rho_{H}(B+D, D+B)=\rho_{H}(A+D, C+B) \\
= & \|[A+D, B+C]\|=\|[A, B]+[D, C]\| \\
= & \|[A, B]-[C, D]\|,
\end{aligned}
$$

that is,

$$
\bar{\rho}([A, B],[C, D]) \leq\|[A, B]-[C, D]\| .
$$

By (6.2) and (6.3), $\bar{\rho}=\rho_{\|\cdot\|}$ and thus $\bar{\rho}$ is a metric. Consequently, $\bar{\rho}$ satisfies the triangle inequality. In view of Lemma 2.5 , this completes the proof.

As a direct consequence of Theorem 6.1, we obtain the following.

Corollary 6.2 Under the assumptions of Theorem 6.1, the function $\hat{\rho}$ is a metric.

Theorem 6.1 combined with Lemma 2.6 yield the following.

Corollary 6.3 If a metric $\rho^{\prime}$ is metrically equivalent to the taxi metric $\rho$ in $\left(\mathcal{K}^{n}, \rho_{H}\right) \times$ $\left(\mathcal{K}^{n}, \rho_{H}\right)$, then the quotient metric $\hat{\rho}^{\prime}$ is metrically equivalent to $\hat{\rho}$.

Open Access This article is distributed under the terms of the Creative Commons Attribution Noncommercial License which permits any noncommercial use, distribution, and reproduction in any medium, provided the original author(s) and source are credited.

\section{References}

Burago, D., Burago, Yu., Ivanov, S.: A course in metric geometry. In: Graduate Studies in Mathematics. AMS (2001)

Gruber, P.M., Lettl, G.: Isometries of the space of convex bodies in Euclidean space. Bull. Lond. Math. Soc. 12, 455-462 (1980)

Herburt, I., Moszyńska, M.: On metric products. Colloq. Math. 92(1), 121-133 (1991)

Herburt, I., Moszyńska, M.: Optimal isometries for a pair of compact convex subsets of $\mathrm{R}^{n}$. In: Convex and Fractal Geometry. Banach Center Publications, vol. 84, pp. 111-120 (2009)

Liapin, E.S.: Semigroups. Translations of Math. Monographs, vol. 3. AMS (1974)

Moszyńska, M.: Selected Topics in Convex Geometry. Birkhäuser (2006)

Pallaschke, D., Urbański, R.: Pairs of compact convex sets. Kluwer, Dordrecht (2002)

Schneider, R.: Isometrien des Raumes der konvexen Körper. Colloq. Math. 33, 219-224 (1975)

Schneider, R.: Convex Bodies: The Brunn-Minkowski Theory. Cambridge University Press (1993)

Volčič, A.: Random symmetrizations of measurable sets. Calc. Var. PDE (2012, to appear) 